\title{
The Reliability of GaN-based LED
}

\author{
Hai Kuang ${ }^{1}$ *, Shi-an $\mathrm{He}^{2, b}$ \\ ${ }^{1}$ Jiangxi Science and Technology Normal University, Nanchang, Jiangxi, \\ 330038, China \\ 2Jiangxi Changyun Company, Nanchang, Jiangxi, 330038, China \\ ahaizi411@126.com, b157898982@qq.com
}

Key word: LED reliability; current aging method; light output; epoxy resin

Abstract: The service life of the LED was much shorter than expected in theory. Its reliability needs to be improved. In this paper, the reliability of the blue GaN-based LED was studied by the current aging method. The light output of LED with different aging time was tested. The results showed that the light intensity decreased faster than that of light power. It may be ascribe to the attenuation of epoxy resin. So the optimal packaging materials were helpful to the improvement of the performance of the LED.

\section{Introduction}

The life of LED is over 100000 hours in theory, but in fact, the service life of the LED is far shorter than this. The reliability of the LED needed to be improved and it aroused extensive attention [1-4]. Electrotechnical Laboratory [5] reported the loss of radiation recombination centers caused by defects was mainly contributed to light droop. Shinji Saito[6] thought the appropriate quantum well depth was helpful to improve the light efficiency. Daniel L.Barton[7] studied the reliability of LED in the process of aging experiment and found that failure of LED was related to carbonization of metal contact and packaging materials. Li Xufeng found that the performance of epoxy resin affected the reliability of the LED directly, so as the light output. The research resultshown by Takeshi Yanagisawa [8] indicated that the life of LED under the pulse current work was 2-4 times longer than that under direct current (DC). G. Meneghesso[9] found large current and high temperature led to the aging of epoxy resin which was in contact with the device, and a layer of opaque layer was formed on the device surface. The reliability of the LED has been widely attracted the attention, but the exact cause for decrease of light output were not clearly.

Under the normal condition, it is difficult to study the reliability of the LED in short time. So the method of accelerated aging was used, which is usually current accelerated aging and temperature accelerated aging. In this paper, the reliability of blue GaN-based LED was studied with the current accelerated aging method. The light output in different aging time was measured.

\section{Experiment}

24 blue GaN-base LEDs with similar parameters were divided into three groups. They were put under constant direct current of $20 \mathrm{~mA}, 30 \mathrm{~mA}, 50 \mathrm{~mA}$ at room temperature, respectively. The light output of LED in the process of testing was tested. The value was the average of every group. The LEDs were packaged with round tips, which were $5 \mathrm{~mm}$ and mainly made of epoxy resin.

\section{Results and discussion}

Light output is one of the important parameters of LED, and is related with the chip type, chip shape and chip size. It is usually represented by luminous intensity and light power. Luminous intensity is the luminous flux in a unit steradian solid angle. It changes with the shape of LED. It is also related with angular direction. The luminous intensity in this paper was of the normal direction. Light is the sum of total radiated power of light, including all wave.

The light power of the GaN-based blue LED changing with the increasing aging time was tested. Fig.1 was shown the normalized (based on the initial value before aging) result with different aging 
time. As can be seen from the Fig.1, light power increased with increasing aging time in 0-24 hours. But after 24 hours, light power gradually decreased. This was contributed to the recombination rate of electrons. At the beginning of aging test, $\mathrm{Mg}-\mathrm{H}$ compounds in the active layer were decomposed and the $\mathrm{Mg}^{+}$icons were released. So the light output was increased, due to the increasing recombination rate[10]. But it attenuates after $24 \mathrm{~h}$. It was reported that may be ascribed to the increased non-radiative recombination centers. The recombination rate deduced and the light output decreased. W.Y. Ho[11] thought it depended on the defects in chip. It was found in their accelerated test that the defect density rose from $2.7 \times 10^{13} \mathrm{~cm}^{-3}$ to $4.2 \times 10^{13} \mathrm{~cm}^{-3}$ where was apart from the conduction band $1.1 \mathrm{eV}$. These defects were considered to be the result of decomposition of $\mathrm{Mg}-\mathrm{H}$ compounds in the process of aging. In addition, some defeat was due to lattice mismatch. Many carriers were captured by these defects. So the light out decreased. Moreover, the non- radiative recombination would lead to the movement and increase number of defects [12]. In the process of aging, the ohmic contact in LED would become worse, leading the attenuation of light output [13], because the metal in ohmic contact would move to the pin area along the defects. The defects in crystal increased in responding [14]. Furthermore, the temperature of LED rose in the process of aging, which would lead to the serious decline on transparency of the epoxy resin, even carbonization of packaging materials[15]. That seriously affected the LED's light output.

It can be seen from Fig.1 that the light power declined more quickly with larger current, ascribing to the more defects. As shown in Fig.1, the light output was always the biggest under 30 $\mathrm{mA}$ and the least under $50 \mathrm{~mA}$. When the current was small, the number of carriers increased with the increasing injection current, so as the recombination rate of electronic-hole. Then the light output increased. When the current increased to $50 \mathrm{~mA}$, carrier number would also increase gradually, but the LED would be destroyed in the big electrical flow with the increase of aging time. In the active layer, there were more defects, which were the non-radiative recombination center. Some electrons and holes were captured in defects. The low radiative recombination rate of the electronic-hole reduced the amount of effective compound, so as the light output. Furthermore, ohmic contact would become worse under high current, which would also lead to weakening of light output.



Fig.1 Light power in process of aging under different current

Fig. 2 was shown the luminous intensity and light power under different currents after being normalized, which was based on the initial value before aging. As can be seen from the Fig. 2, the light power and luminous intensity declined with the increased aging time. This was ascribed to the increasing defects and LED would be worse with long aging time. But the luminous intensity decreased faster than light power. We thought the main reason was the change of the LED light emitting angle of LED. .In the process of aging, the increasing number defects led to destroying of 
epoxy resin. And the high temperature with long aging time was also contributed to the response of epoxy resin. So epoxy resin became yellow even black. The refractive index was changed with the changed color in according to optical law. So the emitting angles changed. Then the light originally emitted from the normal direction changed to the other direction. So the light from the normal direction was less and the light luminous intensity decreased. Light power calculated the light in all directions. So the light power didn't change obviously with the changed emitting angle. As indicated in Fig.2, the light power was stayed above 90\%, but the luminous intensity was below $80 \%$ after $96 \mathrm{~h}$. The luminous intensity was only about $63 \%$ when in aging process for $96 \mathrm{~h}$ under 50 $\mathrm{mA}$. So the driving current should be suitable.
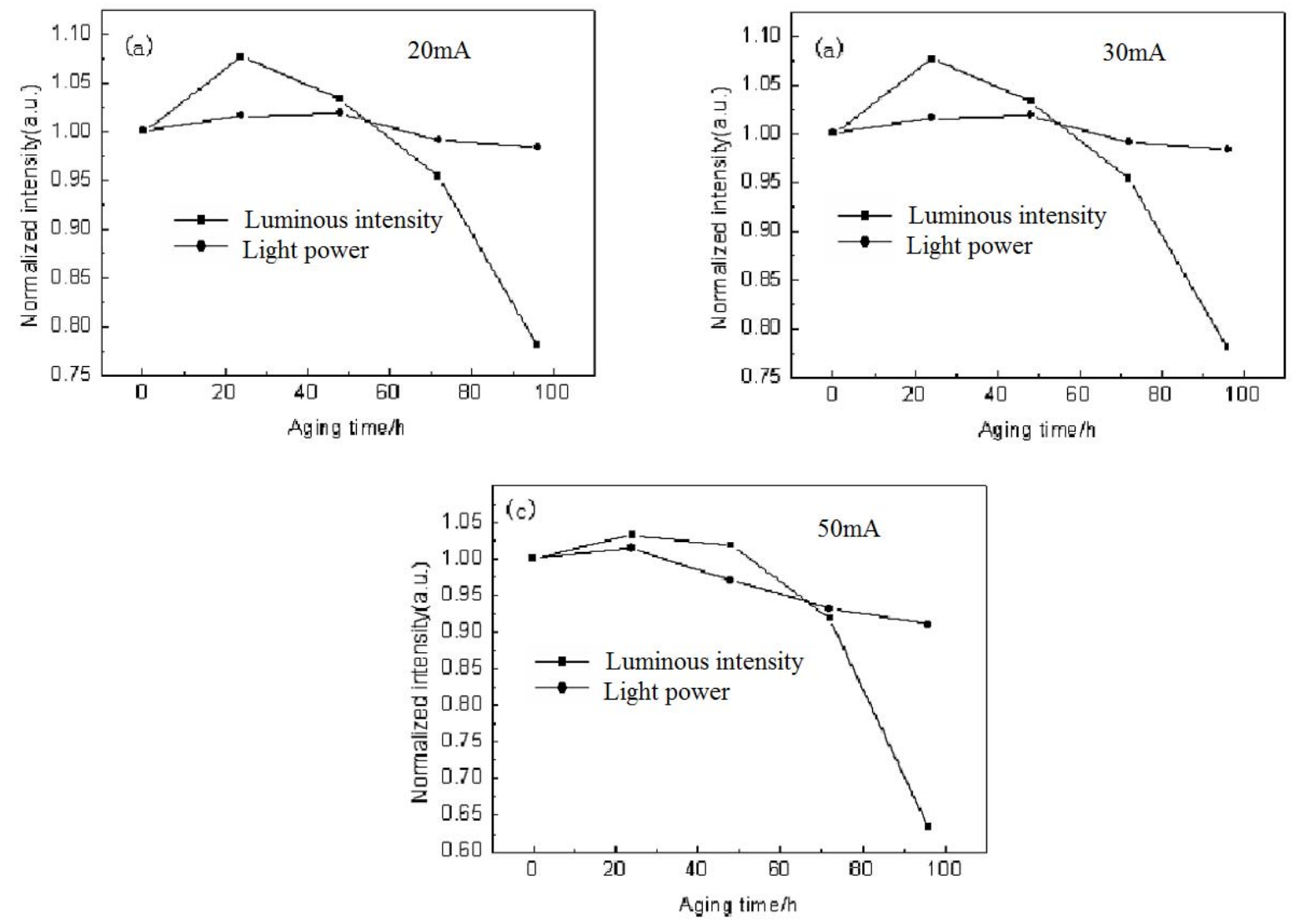

Fig.2 Light output in process of aging under different current

\section{Conclusion}

The reliability is very important for LED. The life of LED is not so long at present. In order to improve the reliability of LED, a current aging experiment was carried out for the blue GaN-based LEDs. The light output was tested. The results showed that:

1. In the process of aging, the LED light power and luminous intensity changed with the creasing aging time. The light power increased at the beginning and decreased after $24 \mathrm{~h}$. It declined fast under $50 \mathrm{~mA}$.

2. The light power was above $90 \%$ after $96 \mathrm{~h}$, indicating the chip was good. But the luminous intensity was below $80 \%$. It was ascribed to the change of emitting angles, due to the defects and damage of LED in high temperature.

3. The appropriate packaging material and suitable driving current is important for reliability of LED.

\section{References}

[1] Hyunsoo Kim, Ji-Myon Lee, Chul Huh, etal.. Phys. Lett., Vol. 77(2000), p.1903-1904

[2] T.Egawa, H.Ishikawa, M.Umeno, App1. Phys. Lett., vol.69 (1996), p.5 
[3] G. Meneghesso, S. Levada, E. Zanoni, et al.. Phys. Stat. So1., Vol.194 (2002), p.389.

[4] N. Narendran, Y.Gu, J. P. Freyssinier, et al. Journal of Crystal Growth, Vol.268 (2004), p. 449-456.

[5] Tsukuba-Shi. Microelectron.Reliab., Vol.37 (1997), p. 1239-1241

[6] Shinji Saito, Masaaki Onomura, Johji Nishio, et a1.. J.Crystal Growth, Vol.189-190,(1998),p.128-132.

[7] Daniel L. Barton, Marek Osinski, Piotr Perlin, et a1.. Microelectronics Reliability, Vol.39(1999),p.1219-1227.

[8] Takeshi Yanagisawa, Takeshi Kojima. Microelectronics Reliability, Vol.43(2003),p.977-980.

[9] G. Meneghesso, et al.. Microelectronics Reliability, Vol.43(2003),p.1737-1042.

[10] F. Manyakhin, A. Kovalev, A. E.Yunovich. MRE Internet Journal of Nitride Semiconductor Research, Vol.3(1998),p.53.

[11] W. Y. Ho, Charlcs Yurya, et al.. IEEE Traps on Electron Devices. Vol.47(7)(2000),p.1421-1425.

[12] Zheng Daishun, Qian Keyuan, Luo Yi. Semiconductor Optoelectronic, Vol. 26(2)(2005),p.87-127.

[13] Delage S L, Dua C. Microelectronics Reliability, Vol.43(2003),p.1705-1712.

[14] H yunsoo Kim, H yundoek Yang, Chul Huh. Electronics Letters, Vol. 36(10)(2000),p.908-910.

[15] L.Barton, Marek Osin'ski, Piotr Perlin, et al. IEEE Proc of the 36th Reliability Physics Symp.New Jersey, (1998),p.119-123. 